\title{
The immune complex p53 protein/anti-p53 autoantibodies in the pathogenesis of ovarian serous carcinoma
}

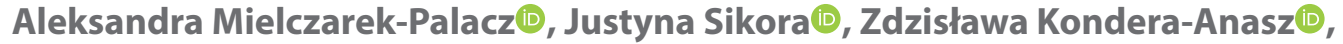 \\ Marta Smycz-Kubańska®D, Aleksandra Englisz®D, Jarosław Strzelczyk, Jacek Kabut
}

Department of Immunology and Serology, Faculty of Pharmaceutical Sciences in Sosnowiec, Medical University of Silesia, Katowice, Poland

\begin{abstract}
Objectives: The tests conducted were intended to analyze the concentration of p53 protein and anti-p53 autoantibodies in serum of women with ovarian tumours.

Material and methods: The study included patients with diagnosed ovarian cancer: Cystadenoma serosum or Cystadenocarcinoma papillare serosum at Illc stage (including 10 women who had G1, 14 women who had G2 and 30 women who had G3 staging). Concentrations of parameters were measured by ELISA.

Results: The analysis of the obtained results showed statistical significance between the concentration of p53 protein depending on the degree of differentiation of $\mathrm{G} 1$ and $\mathrm{G} 3(\mathrm{p}<0.001)$ and anti-p53 autoantibodies depending on the degree of differentiation of $G 1$ and $G 2(p<0.05)$ as well as $G 2$ and $G 3(p<0.01)$. In addition, the determined p53/anti-p53 autoantibodies ratio was only significant between $\mathrm{G} 1$ and G2 $(p<0.05)$, as was the assessment of the percentage of the tested parameters in the immune complex.

Conclusions: Immune system disorders involving the p53 protein and anti-p53 autoantibodies may be one of the immune mechanisms involved in the pathogenesis of ovarian serous cancer.
\end{abstract}

Key words: autoantibodies anti-p53/ p53 protein; ovarian cancer

Ginekologia Polska 2020; 91, 9: 519-523

\section{INTRODUCTION}

In the course of ovarian carcinoma, an important role plays the immune response directed against neoplastic cells, especially apoptosis. It is one of the key processes leading to initiation of carcinogenesis [1].

In the regulation of apoptosis, a significant role is also performed by protein $\mathrm{p} 53$, which participates in the intrinsic pathway of the process, called a p53-dependent mitochondrial pathway. Protein p53, encoded by the gene TP53 and localized on chromosome 17p13.1, is crucial for a proper course of the cell cycle and therefore, is called a guardian of the genome. One of the major functions of the $\mathrm{p} 53$ protein is to activate transcriptions of genes responsible for initiating apoptosis in response to DNA damage, which may be linked to its anti-tumour activity $[2,3]$. The loss of protein $p 53$ activity was shown in the pathogenesis of many neoplasms, while the most often mechanism leading to this phenomenon is the point mutation of the gene TP53 $[4,5]$.
Mutations in TP53 head for creating a protein with a decreased activity, or a native protein which is not capable of regulating the cell cycle and effectively fulfilling its function $[6,7]$. The mutated p35 protein supports the survival and proliferation of neoplastic cells, inhibits their apoptosis, and promotes resistance to chemotherapy. The altered form of the protein has a longer half-life in the organism, which enables its determination in the extracellular fluid, including blood serum [8].

The studies concerning ovarian neoplasms conducted so far also indicate disturbances in humoral immune response manifesting itself in production of autoantibodies anti-p53 $[9,10]$. Detection of anti-p53 autoantibodies in biological fluids are the result of the secondary humoral response directed against the mutated protein $\mathrm{p} 53$. They belong to antibodies of IgG class and may be detected even after several years from diagnosing a neoplastic change $[11,12]$. 
Due to the fact that the systemic changes accompanying ovarian neoplasms and occurring with soluble apoptosis mediators are still not fully known, their fuller evaluation requires further studying. Taking into the account that these changes may perform a crucial function in the pathogenesis of ovarian cancers and that the concentration of the immune system soluble mediators in body fluids may be applied in clinical practice, the primary objective of the paper was to assess the concentration of p53 protein and anti-p53 autoantibodies in the serum of women with ovarian tumours.

\section{MATERIAL AND METHODS}

This study had 74 women aged 23 to 70 years (mean age: $37.5 \pm 9.42$ years), who were diagnosed with ovarian cancer, qualified for the study. In this group, 20 women had Cystadenoma serosum and 54 women had Cystadenocarcinoma papillare serosum at IIIc stage according to the FIGO classification and, within the group, 10 patients were diagnosed with $G 1,14$ were diagnosed with $G 2$, and the remaining 30 were diagnosed with $\mathrm{G} 3$ staging. Clinical diagnosis of the tumour was confirmed by histopathological examination. Any other reproductive system disease was not found in the examined women. The patients were hospitalized in the Clinical Ward of Obstetrics and Gynecology, Women's Health Chair, Medical University of Silesia in Ruda Śląska. The control group included 34 healthy women aged between 27-60 (mean age: $45.34 \pm 19.25$ years). No pathological changes in these women's reproductive organs occurred. The research material in all the women was the blood serum. The blood was taken from women after making clinical diagnosis, before surgery. In the morning the blood was taken from the cubital vein, to a clot tube, in order to obtain the serum and 30 minutes after taking the blood, it was centrifuged at $1500 \times \mathrm{g}$ for 15 minutes. Until testing was performed, the serum was stored at $-80^{\circ} \mathrm{C}$. In the control group, the blood was taken while the women came for the check-up and the same procedure was used. Enzyme-linked immunosorbent assay (ELISA) was used to determine the concentration of the studied parameters: p53 Kit Elisa Gen-Probe, Diaclone and anti-p53 autoantibodies ELISA, Steinbeis-Transferzentrum, Angewandte Biologische Chemie. Test sensitivity was the following: $1.5 \mathrm{U} / \mathrm{mL}$ (for p53) and $0.07 \mathrm{U} / \mathrm{mL}$ (for anti-p53 autoantibodies). All the women, who participated in the study, consented to conducting the research. The approval of the Ethics Committee of the Medical University of Silesia in Katowice was obtained.

Using the computer program Statistica for Windows 10.0 and Microsoft Excel, the results were statistically analyzed. The Shapiro-Wilk test was used in order to verify the distribution of the obtained results. After verification that the received results corresponded to the normal distribution, the arithmetic mean ( $\mathrm{x}$ ) and standard deviation (SD) were calculated for each parameter. The mean values of the studied parameters in the studied group and the control group were compared by means of the Student's t-test, while for nonparametric results: Mann-Whitney's test was used. Correlations were tested by Spearman's rank correlation test and presented as correlation coefficient ( $r$ ). $P<0.05$ was considered statistically significant.

\section{RESULTS}

In the serum of healthy women belonging to the control group and women with ovarian serous cystadenoma, the concentration of the p53 was below the test sensitivity threshold which amounted to $1.5 \mathrm{U} / \mathrm{mL}$. In the group of women with ovarian cancer, due to the fact that the obtained values did not correspond with the normal distribution, the results are presented as a median and an upper and lower interquartile range (Q1 and Q3) which equalled $22.14 \mathrm{U} / \mathrm{mL}$ and $47.71 \mathrm{U} / \mathrm{mL}$, respectively, while the median value was equal to $35.89 \mathrm{U} / \mathrm{mL}$. The conducted analysis of the test results revealed statistical significance between the concentration of the studied parameter depending on the degree of differentiation $\mathrm{G} 1$ and $\mathrm{G} 3(\mathrm{p}<0.001)$. However, no significant differences between the concentration of p53 protein in the serum of women with moderately differentiated (G2) and poorly differentiated (G3) ovarian cancer were shown. The obtained results are shown in Figure 1. There was no correlation between CA125 concentration in the serum of women with ovarian cancer and the concentration of $\mathrm{p} 53$ protein in their serum.

Further analysis included the assessment of anti-p53 autoantibodies concentration in the studied women. In the serum of healthy women and women with ovarian serous cystadenoma, anti-p53 autoantibodies were not detected. Whereas in the group of women with ovarian cancer concentration of the parameter did not correspond with the

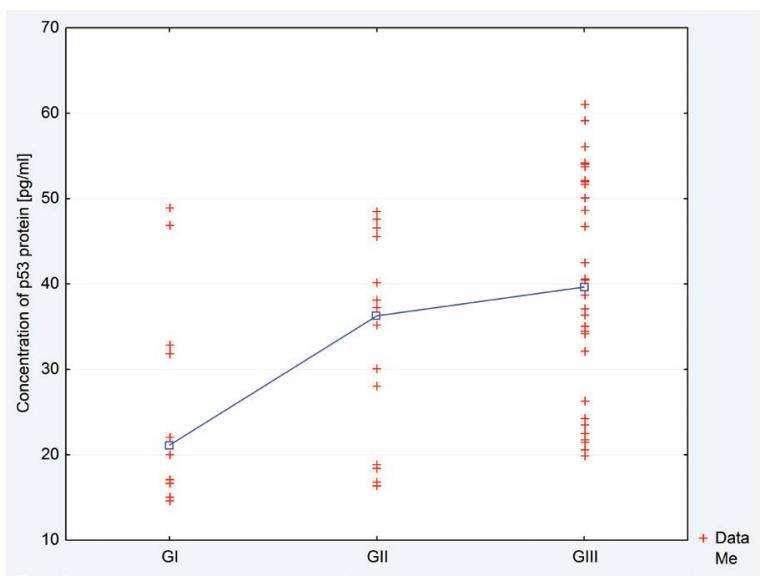

Figure 1. Concentrations of $\mathrm{p} 53$ protein in serum of women depending on the degree of differentiation G1, G2 and G3 


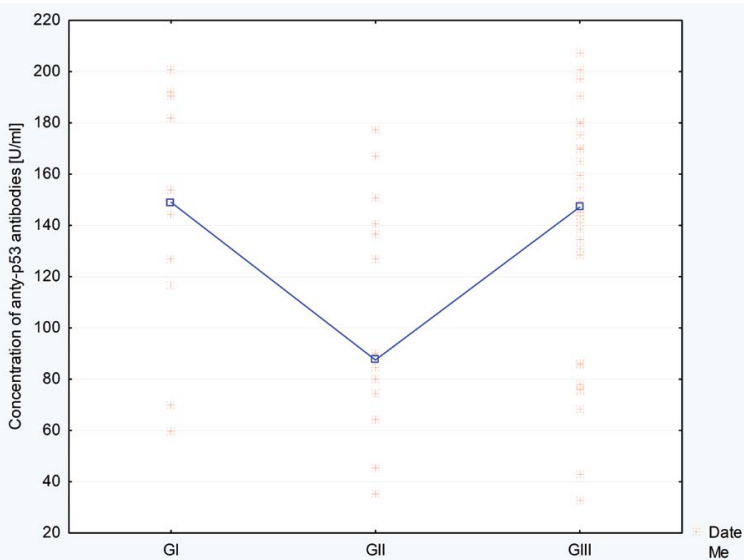

Figure 2. Concentrations of anti-p53 autoantibodies in serum of women depending on the degree of differentiation G1, G2 and G3

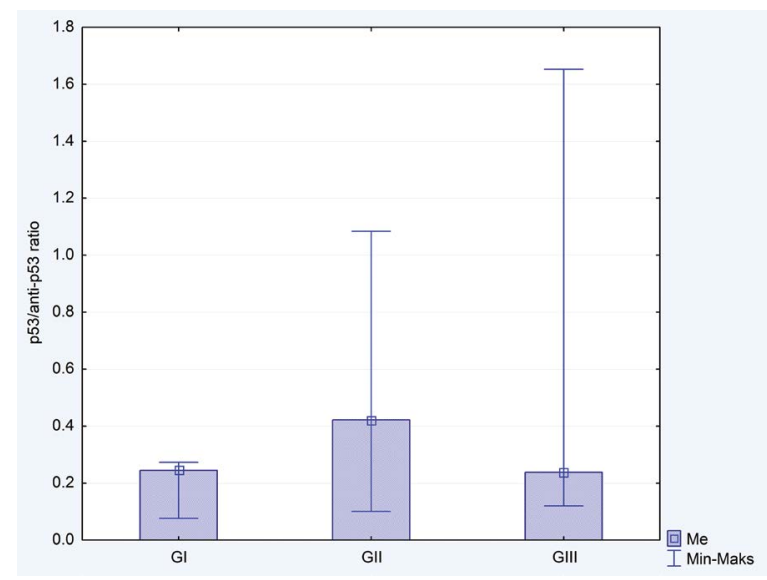

Figure 3. p53/anti-p53 autoantibodies ratio in serum of women depending on the degree of differentiation $\mathrm{G} 1, \mathrm{G} 2$ and $\mathrm{G} 3$

normal distribution and therefore the results are presented as a median and an upper and lower interquartile range (Q1 and Q3), amounted to $80.30 \mathrm{U} / \mathrm{mL}$ and $170.10 \mathrm{U} / \mathrm{mL}$ respectively, while the median value equalled $140.92 \mathrm{U} / \mathrm{mL}$. The conducted analysis of the study results revealed a significance between the concentration of the studied parameter depending on the degree of differentiation $\mathrm{G} 1$ and $\mathrm{G} 2(\mathrm{p}<0.05)$ and $\mathrm{G} 2$ and $\mathrm{G} 3(\mathrm{p}<0.01)$. The obtained results are shown in Figure 2. There was no correlation between the concentration of CA125 in the serum of women with an ovarian neoplasm and anti-p53 autoantibodies concentration in their serum.

Subsequently, the ratio p53/anti-p53 autoantibodies was determined. A statistically significant difference was shown for the value of this coefficient only between $\mathrm{G} 1$ and $\mathrm{G} 2(\mathrm{p}<0.05)$ in Figure 3.

Moreover, since in the serum of women with ovarian cancer, the concentration anti-p53 autoantibodies was

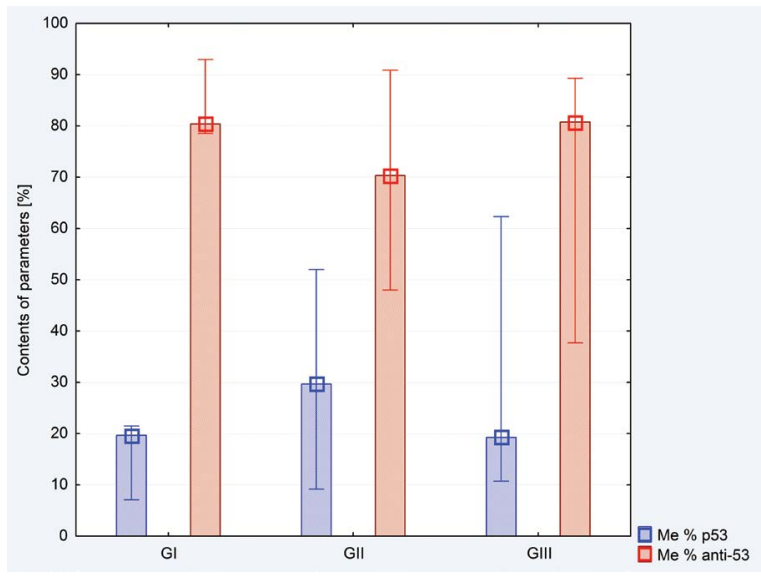

Figure 4. Percentage contents of $\mathrm{p} 53$ protein and anti-p53 autoantibodies in serum of women depending on the degree of differentiation $\mathrm{G} 1, \mathrm{G} 2$ and $\mathrm{G} 3$

three times higher than the concentration of protein $\mathrm{p} 53$, the percentage contents of protein $\mathrm{p} 53$ and autoantibodies p53 in the total immune complex was determined. The analysis revealed a statistically significant increase of the percentage contents of 553 protein in the total immune complexes in the serum between $\mathrm{G} 1$ and $\mathrm{G} 2$, was illustrated in Figure 4.

\section{DISCUSSION}

Studies have so far indicated that some neoplastic cells may reveal sensitivity to factors inducing the intrinsic pathway of apoptosis and therefore, it is justifiable to study the markers of this process. It is thanks to its apoptosis conduct that the neoplastic fully transformed cells are eliminated. The imbalance between the number of proliferating cells and those undergoing apoptosis may be caused by the presence of the p53 protein. It influences the decrease of ability to activate the programmed death of the cell which is irreversibly damaged. Its presence may also lead to initiation of the neoplastic process. The altered form of the protein has a longer half-life in the organism which enables its determination $[13,14]$.

The previous studies concerned mostly the assessment of protein p53 expression in women with ovarian cancer using the immunohistochemical method [15], whereas in this study we tried to assessment of the concentration of this parameter in the serum of women with ovarian cancer.

In our studies, the concentration of protein p53 in the serum of both women with ovarian cancer and from the control group was assessed. The analysis included 74 female patients with a diagnosed serous ovarian cancer at the IIIC stage according to FIGO classification and with ovarian serous cystadenoma. In the serum of healthy women constituting the control group and women with serous 
cystadenoma, the concentration of p53 protein was below the sensitivity threshold. However, a significantly increased concentration of protein p53 in the serum of women with ovarian cancer when compared to its concentration assessed in the control group was indicated. The obtained increased concentration of protein p53 points out to impairment of the apoptotic process on the intrinsic pathway. Interesting was the analysis of the concentration of the studied parameter conducted according to the degree of tumour differentiation. The indicated statistical significance between $\mathrm{G} 1$ and $\mathrm{G} 3$ proves that the impaired apoptosis with protein p53 is associated with the degree of histological differentiation of ovarian cancer.

Very little data are currently available on the prevalence of anti-p53 antibodies in patients with epithelial ovarian cancer [16]. Murphy et al. [17] used ELISA to detect anti-p53 antibody in sera in normal heath individuals and ovarian cancer patients, however, the distribution of anti-p53 antibody in the two groups showed no significant difference. Additionally, Høgdall et al. [18] found that the frequency of anti-p53 antibodies in ovarian cancer patients increased somewhat with increasing clinical stage of disease, but the differences did not reach statistical significance. In contrast, other studies showed opposite results [19-21]. Anti-p53 antibodies are often detected in serum from patients with advanced ovarian carcinoma [22]. Additionally, the preoperative serum anti-p53 antibody status had no prognostic relevance for progression-free survival and survival [23]. However, the generation of a humoral immune response against p53 protein in the close tumour environment, as demonstrated by the occurrence of anti-p53 autoantibodies in the ascitic fluid of ovarian carcinoma patients, is associated with poor disease-free survival [24]. Many researchers have been interested in the use of autoantibodies as serological markers for cancer diagnosis, because of the absence of these autoantibodies in normal individuals and in non-cancer conditions. With the successive addition to study panel, except p53, another autoantigen tumour associate to (surviving, p16, cyclin B1, cyclin D1, cyclin A and cyclin E), there was a stepwise increase in sensitivity of up to $62.5 \%$, and in specificity of $90.2 \%$ [19].

Currently, there are many studies conducted aiming at determination of a possible application of anti-p53 autoantibodies in the diagnostics of ovarian cancer. Arjomandi et al. [25] presented an innovative algorithm which could increase the specificity and sensitivity of potential tests for early detection of ovarian cancer. For detection, the authors used the protein p53 complex with selected antigenic epitopes. Based on the conducted analyses, they did not demonstrate the determination of the studied autoantibodies in the screening diagnostics of ovarian cancer. Moreover, according to the authors, assessing the concentration of anti-p53 autoantibodies with CA125 does not constitute a sufficiently sensitive and specific screening test for detection of early lesions in female patients with ovarian cancer.

Anderson et al. [20] observed a significantly increased concentration of autoantibodies in the serum of over $42 \%$ of women with serous ovarian cancer, whereas in the case of different histological types of cancer as well as benign tumours, the concentration of antibodies was considerably decreased. In the opinion of researchers, the application of anti-p53 autoantibodies as markers for early detection of ovarian cancer is limited due to a low sensitivity at an early stage of disease and non-specific reactions in the case of neoplasms demonstrating an increased expression of abnormal protein $\mathrm{p} 53$.

The assessment of autoantibodies p53 application in monitoring the efficacy of the basic treatment therapy of ovarian cancer was also made by Häfner et al. [26]. The authors demonstrated that antibodies p53 could constitute a more sensitive marker than antigen CA 125 in detecting minimal residual disease remaining after the surgical procedure or chemotherapy. The concentration of antigen CA125, released by neoplastic cells, is associated with the mass of ovarian tumours, while the induction of antibodies $\mathrm{p} 53$ takes place in response to the occurrence of single neoplastic cells. According to the authors, assessing p53 antibodies could become useful in monitoring the maintenance and consolidation therapy.

Similarstudies were performed outby Dobrzycka etal.[27], who found antibodies p53 in 33,3\% of female patients undergoing aggressive treatment of ovarian cancer. A significantly increased concentration of these antibodies was found in the serum of patients with serous ovarian cancer with III and IV clinical stage of tumour. Moreover, as a part of the conducted analysis in female patients with serous cancer anti-p53 autoantibodies positive, they assessed the values for the chance of one-year and five-year survival with a diagnosed cancer which amounted to $86.4 \%$ and $72.7 \%$ respectively. The median of the overall survival time for women anti-p53 autoantibodies positive was equal to 11 months, while for patients anti-p53 autoantibodies negative, it amounted to 57 months. In the case of women with mucinous cancer they did not demonstrate the correlation between the presence of the determined antibodies in the serum, and the overall survival time [27].

Our date did not demonstrate the presence of antibodies p53 in the serum of healthy women and women with serous cystadenoma. However, there was found a significantly increased concentration of anti-p53 autoantibodies complex in the serum of women with ovarian cancer.

Interestingly, the determined coefficient p53/anti-p53 autoantibodies which was almost 3-times higher in women with ovarian cancer than in the control group, 
may indicate impairments in the internalization of the immune complex occurrence in the system which in turn undoubtedly favours the induction of the immune tolerance in response to the growing tumour. On the other hand, the conducted assessment of the percentage contents of p53 protein and anti-p53 autoantibodies in the immune complex proves that patients with ovarian cancer are accompanied by the impairment of immunoprecipitated p53 protein - anti-p53 autoantibodies occurrence, while the excess of free antibodies in the serum may have an influence on a weaker immune reactivity by interfering with some soluble mediators of the immune system. In contrast, the increased levels of p53-AAbs in the serum of women with ovarian cancer indicate an increased effect of secondary humoral response against the mutated p53 protein, and they are also associated with the degree of histological differentiation of the tumour, and their determination may be used in laboratory diagnostics, which requires, however, further research.

\section{CONCLUSIONS}

The results of the conducted studies allow to draw the following conclusions:

1. The regulation of the immune response towards suppression and the creation of an environment conducive to the proliferation of cancer cells may involve disorders associated with the $\mathrm{p} 53$ protein/anti-p53 autoantibodies system.

2. The occurring systemic changes in the immune system with soluble mediators play an important role in the pathogenesis of ovarian cancer. Intensification of apoptosis and humoral immune response impairment in women with ovarian cancer confirms a considerable involvement of $\mathrm{p} 53$ protein and anti-p53 autoantibodies in the pathogenesis of serous ovarian cancer.

\section{REFERENCES}

1. Turner TB, Buchsbaum DJ, Straughn JM, et al. Ovarian cancer and the immune system - The role of targeted therapies. Gynecol Oncol. 2016; 142(2): 349-356, doi: 10.1016/j.ygyno.2016.05.007, indexed in Pubmed: 27174875.

2. Choschzick M, Hantaredja W, Tennstedt P, et al. Role of TP53 mutations in vulvar carcinomas. Int J Gynecol Pathol. 2011; 30(5): 497-504, doi: 10.1097/PGP.0b013e3182184c7a, indexed in Pubmed: 21804386.

3. Amaral JD, Xavier JM, Steer CJ, et al. The role of p53 in apaptosis. Discov Med. 2010; 9: 145-52.

4. Berkers CR, Maddocks ODK, Cheung EC, et al. Metabolic regulation by p53 family members. Cell Metab. 2013; 18(5): 617-633, doi: 10.1016/j. cmet.2013.06.019, indexed in Pubmed: 23954639.

5. Yue $X$, Zhao $Y, X u Y$, et al. Mutant p53 in Cancer: Accumulation, Gain-of-Function, and Therapy. J Mol Biol. 2017; 429(11): 1595-1606, doi: 10.1016/j.jmb.2017.03.030, indexed in Pubmed: 28390900.

6. Yang X, Jiang P, Du W. p53 and regulation of tumor metabolism. Journal of Carcinogenesis. 2013; 12(1): 21, doi: 10.4103/1477-3163.122760.

7. Liu J, Zhang C, Feng Z, et al. Tumor suppressor p53 and its mutants in cancer metabolism. Cancer Lett. 2015; 356(2 Pt A): 197-203, doi: 10.1016/j.canlet.2013.12.025, indexed in Pubmed: 24374014.
8. Zhang J, Xu Z, Yu L, et al. Assessment of the potential diagnostic value of serum p53 antibody for cancer: a meta-analysis. PLoS One. 2014; 9(6): e99255, doi: 10.1371/journal.pone.0099255, indexed in Pubmed: 24911057.

9. Wu M, Mao C, Chen Q, et al. Serum p53 protein and anti-p53 antibodies are associated with increased cancer risk: a case-control study of 569 patients and 879 healthy controls. Mol Biol Rep. 2010; 37(1): 339-343, doi: 10.1007/s11033-009-9744-7, indexed in Pubmed: 19693693.

10. Suppiah A, Greenman J. Clinical utility of anti-p53 auto-antibody: systematic review and focus on colorectal cancer. World J Gastroenterol. 2013; 19(29): 4651-4670, doi: 10.3748/wjg.v19.i29.4651, indexed in Pubmed: 23922463.

11. Chatterjee M, Tainsky MA. Autoantibodies as biomarker for ovarian cancer. Cancer Biomark. 2010; 8: 187-201.

12. Garziera M, Montico M, Bidoli E, et al. Prognostic Role of Serum Antibody Immunity to p53 Oncogenic Protein in Ovarian Cancer: A Systematic Review and a Meta-Analysis. PLoS One. 2015; 10(10): e0140351, doi: 10.1371/journal.pone.0140351, indexed in Pubmed: 26451959.

13. Lu D, Kuhn E, Bristow RE, et al. Comparison of candidate serologic markers for type I and type Il ovarian cancer. Gynecol Oncol. 2011; 122(3): 560-566, doi: 10.1016/j.ygyno.2011.05.039, indexed in Pubmed: 21704359.

14. Brachova $\mathrm{P}$, Thiel KW, Leslie KK. The consequence of oncomorphic TP53 mutations in ovarian cancer. Int J Mol Sci. 2013; 14(9): 19257-19275, doi: 10.3390/ijms140919257, indexed in Pubmed: 24065105.

15. Gajewska M, Wielgoś M, Panek G, et al. Incidence of proapoptotic proteins p53 and p21 in epithelial ovarian tumors. Ginekol Pol. 2014; 85(2): 111-116, doi: 10.17772/gp/1700.

16. Shi JX, Qin JJ, Ye H, et al. Tumor associated antigens or anti-TAA autoantibodies as biomarkers in the diagnosis of ovarian cancer: a systematic review with meta-analysis. Expert Review of Molecular Diagnostics. 2015; 15(6): 829-852, doi: 10.1586/14737159.2015.1035713.

17. Murphy MA, O'Connell DJ, O'Kane SL, et al. Epitope presentation is an important determinant of the utility of antigens identified from protein arrays in the development of autoantibody diagnostic assays. J Proteomics. 2012; 75(15): 4668-4675, doi: 10.1016/j.jprot.2012.02.031, indexed in Pubmed: 22415278.

18. Høgdall EVS, Høgdall CK, Blaakaer J, et al. P53 autoantibodies in sera from Danish ovarian cancer patients and their correlation with clinical data and prognosis. APMIS. 2002; 110(7-8): 545-553, doi: 10.1034/j.160 0-0463.2002.11007805.x, indexed in Pubmed: 12390412.

19. Li L, Wang K, Dai L, et al. Detection of autoantibodies to multiple tumor-associated antigens in the immunodiagnosis of ovarian cancer. Molecular Medicine Reports. 2008, doi: 10.3892/mmr.1.4.589.

20. Anderson KS, Wong J, Vitonis A, et al. p53 autoantibodies as potential detection and prognostic biomarkers in serous ovarian cancer. Cancer Epidemiol Biomarkers Prev. 2010; 19(3): 859-868, doi: 10.1158/10559965.EPI-09-0880, indexed in Pubmed: 20200435.

21. Lu D, Kuhn E, Bristow RE, et al. Comparison of candidate serologic markers for type I and type Il ovarian cancer. Gynecol Oncol. 2011;122(3):560-566, doi: 10.1016/j.ygyno.2011.05.039, indexed in Pubmed: 21704359.

22. Gadducci A, Ferdeghini M, Buttitta F, et al. Serum anti-p53 antibodies in the follow-up of patients with advanced ovarian carcinoma. Anticancer Res. 1998; 18(5B): 3763-3765, indexed in Pubmed: 9854491.

23. Gadducci A, Ferdeghini M, Buttitta F, et al. Assessment of the prognostic relevance of serum anti-p53 antibodies in epithelial ovarian cancer. Gynecol Oncol. 1999; 72(1): 76-81, doi: 10.1006/gyno.1998.5101, indexed in Pubmed: 9889034.

24. Abendstein B, Marth C, Müller-Holzner E, et al. Clinical significance of serum and ascitic p53 autoantibodies in epithelial ovarian carcinoma. Cancer. 2000; 88(6): 1432-1437, doi: 10.1002/(sici)1097-0142(200003 15)88:6<1432::aid-cncr22>3.0.co;2-8, indexed in Pubmed: 10717627.

25. Arjomandi A, Delanoy ML, Walker RP, et al. A novel algorithm to improve specificity in ovarian cancer detection. Cancer Treat Res Commun. 2018; 15: 32-35, doi: 10.1016/j.ctarc.2017.11.004, indexed in Pubmed: 30207285.

26. Häfner N, Nicolaus $\mathrm{K}$, Weiss $\mathrm{S}$, et al. p53-autoantibody may be more sensitive than CA-125 in monitoring microscopic and macroscopic residual disease after primary therapy for epithelial ovarian cancer. Journal of Cancer Research and Clinical Oncology. 2013; 139(7): 1207-1210, doi: 10.1007/s00432-013-1432-2.

27. Dobrzycka B, Terlikowski SJ, Kinalski M, et al. Circulating free DNA and p53 antibodies in plasma of patients with ovarian epithelial cancers. Ann Oncol. 2011; 22(5): 1133-1140, doi: 10.1093/annonc/mdq584, indexed in Pubmed: 21098618. 